\title{
LAS CIENCIAS HUMANAS COMO PRACTICAS DISCURSIVAS
}

\section{María del Pilar Britos}

Al recorrer los trabajos de Michel Foucault, especialmente los que pertenecen a su primera etapa - 'Las palabras y las cosas', 'La arqueología del saber', 'El orden del discurso'...- detectamos un sostenido esfuerzo por definir en qué consiste el proyecto arqueológico, a qué se dirige y cómo se distingue de otras perspectivas históricoepistemológicas. El objeto de esta propuesta se va delineando en torno a la categoría de 'prácticas discursivas', mediante la cual Foucault puede orientar el análisis del discurso de las ciencias humanas en su materialidad efectiva. Más 'acá' de la reflexión acerca de las condiciones de validez de las teorías científicas, la arqueología descubre los 'discursos serios' como acontecimientos que producen efectos de verdad a nivel de la interacción social y de la vida de los individuos. Se construyen así una serie de conceptos que permiten explicitar la problemática de la conexión saber-poder en la sociedad moderna e incluso reformular los términos de la relación teoría-práctica.

Lo que queremos plantear aquí no es la urgencia de una sistematización de los términos en que sería posible analizar / clasificar / evaluar los discursos y saberes hoy vigentes. Lo que pretendemos es repensar algunas categorías y relaciones que han sido 'removidas' en los trabajos de este autor y que, en esta nueva perspectiva, nos permitirían reconsiderar algunas problemáticas que se hacen presentes en la reflexión acerca de las ciencias humanas entendidas como 'prácticas discursivas'.

1- La arqueología se plantea, ante todo, como un modo de aproximación a los campos del saber que han incidido, e inciden, en la conformación del sujeto moderno. Podemos entenderla como una mirada retrospectiva que nos permite rastrear los procesos de formación del 'saber' que hoy tenemos como válido. Y no se trata de cualquier saber sino fundamentalmente de 'éste' que hoy nos dicta la verdad 
acerca de nosotros mismos y que constituye el terreno de lo que llamamos ciencias humanas.

La propuesta de análisis no apunta a un nivel trascendental -las condiciones de posibilidad de toda experiencia- sino a una descripción localizada del 'archivo' (1) de aquellas formaciones discursivas que están presentes en la genealogía del sujeto moderno. Se trata entonces de un rastreo, una vuelta atrás para detectar los problemas a los que las ciencias han intentado responder, 0 , dicho de otro modo, para descubrir cuáles han sido las situaciones humanas que se señalaron como 'problemas a resolver' y dieron pie a la estructuración de un saber que las explique, clasifique, comprenda.

En este marco, la noción de 'problematización' constituye el eje que permite articular los procesos históricos de formación del saber. Esto es, a partir de un interrogante que surge como expresión de una necesidad histórica concreta, se conforma una experiencia del objeto en la cual éste entra en el ámbito de distinción de lo verdadero y lo falso, de lo igual y lo diferente, de lo que es y lo que debe ser. Esta visión o percepción del objeto no se puede justificar desde la mera intencionalidad de la conciencia, desde sus categorías o su contexto histórico social, como si éste fuera el origen y aquél el producto. El proceso es más complejo. En la medida en que algo se problematiza -algo vivido, percibido, dicho- se constituye un nuevo campo de visibilidad, se inaugura un nuevo régimen de enunciados que va a provocar una discontinuidad, no sólo a nivel de las significaciones de este objeto sino también respecto de las representaciones de otros objetos colaterales, de las estrategias conceptuales que se empleen, de las posiciones subjetivas posibles. La formación de un saber remite entonces al espacio de interrogación y regulación que posibilita la construcción de un determinado conocimiento como contenido efectivo de una problematización particular.

"Ast, hablar del 'objeto' de una ciencia significa afrontar un problema que primero debe ser planteado y luego resuelto; hablar de

(1) Cf. la noción de 'archivo' en M. Foucault, "La arqueología del saber", Siglo XXI, pág. 214 ss. 
la historia de una ciencia impone mostrar de qué manera -por qué motivos teóricos o prácticos- una ciencia 'se las arreglo' para plantear y resolver ese problema". (2)

Por otra parte, este análisis dejar aparecer también la posibilidad históricamente negada de ciertos discursos que, aunque fueran 'epistemológicamente' posibles, no han tenido cabida en el orden de lo que fue visible y enunciable en una época dada. En este sentido, Foucault introduce en el análisis el 'principio de reinversión' a partir del cual opone al rol 'positivo' de lo que se afirma en una disciplina, el reconocimiento del 'juego negativo' de lo que se recorta y queda fuera de este sistema de enunciación. Entonces, si nos referimos a un 'a priori histórico' de los saberes para señalar las condiciones de su aparición efectiva, es preciso discernir alli mismo las formas de exclusión, limitación, apropiación, coacción que conlleva este proceso de formación discursiva. (3) Se distingue aś la tarea arqueológica del enfoque tradicional de la historia de las ideas, en tanto ya no se busca desentrañar las representaciones que puede haber detrás del discurso, sino descubrir "el juego de reglas que determinan la aparición y desaparición de los enunciados en una cultura". (4)

(2) A. Gabilondo, "El discurso en acción", Ed. Ahthropos, 1990, p. 37

(3) Cf. M. Foucault, "L'ordre du discours", Gallimard, 1971, p. 62 ss.

(4) M. Foucault, "Réponse au Cercle d'Epistémologie", en Cahiers pour l'analyse $\mathrm{N}^{\circ} 9,1968$, p. 19. (La traducción es nuestra).

En otra oportunidad, Foucault aclara ". . .De aquí que la tarea no sea ni una formalización ni una exégesis sino una arqueologia, es decir, la descripción de un archivo". (Réponse à une question, Esprit, 1968). Refiriéndose a estas palabras de Foucault, A. Gabilondo señala: "Han de establecerse, por tanto, los límites y las formas de la decibilidad (de qué es posible hablár y qué es lo que ha sido como dominio del discurso); los límites y las formas de conservación (qué enunciados dejan huella -en la recitación ritual, la pedagogía, la diversión, la publicidad. . .-, cuáles son reprimidos y censurados); los límites y las formas de la memoria tal como aparecen en las diferentes formaciones discursiva (qué enunciados son reconocidos como válidos o discutibles, o definitivamente invalidados, qué tipo de relaciones se han establecido entre los vistemas de enunciados presentes y el corpus de los pasados); los límites y las 
2. Ahora bien, esta aproximación arqueológica no solo avanza en el orden de una historia de las ciencias -de lo que podríamos llamar una 'historia de las problematizaciones' - sino que exige recorrer también horizontalmente el diagrama de relaciones discursivas y no discursivas en que se inscribe el saber acerca del hombre. El arqueólogo ha de ser un "archivista" pero también un "cartógrafo". (5)

Lo que está en la mira del proyecto de Foucault no son los discursos en tanto textos que dan cuenta de su propio orden / organización / coherencia. Tampoco son las prácticas en tanto acciones individuales o colectivas que se deciden a partir de una situación, de un proyecto. A lo que se apunta es a las 'prácticas discursivas', es decir, al espacio de relaciones entre enunciados y medios no - discursivos en el que se hacen visibles las reglas que determinan el juego de posibilidades - e imposibilidades - en el que participan los individuos en un momento dado, en una sociedad.

"Foucault nunca ha buscado elaborar una teoría universal del discurso sino más bien describir las diferentes formas historicas de las prácticas discursivas. (...) Hacer una descripción objetiva de la manera regulada en que se autorrige el discurso rigiendo las prácticas y las instituciones sociales". (6)

Podemos hablar entonces de las ciencias humanas como 'prácticas discursivas', como un 'saber ejercido', un juego de relaciones entre las significaciones formuladas e institucionalizadas, entre las estrategias teóricas y los dispositivos concretos que se deciden y se ponen en

formas de reactivación (qué enunciados anteriores o extranjeros se retienen, se valoran, qué sistema de apreciación se les aplica, qué papel se les hace cumplir. . .); los límites y las formas de la apropiación (qué individuos, qué grupos, qué clases tienen acceso a tal tipo de discurso, cómo se señala y se define la relación del discurso con su autor. . .)". (Op. cit. pág. 123-124).

(5) Ambos términos han sido empleados por G. Deleuze en el libro dedicado a M. Foucault. (Cf. G. Deleuze, 'Foucault', Ed. Minuit, 1986).

(6) H. Dreyfus - P. Rabinow, "Michel Foucault, un parcours philosophique" Gallimard, 1984, p. 9-10. (La traducción es nuestra). 
marcha en un momento histórico preciso: el confinamiento de los locos, el sistema carcelario, los dispositivos de sexualidad... (7)

La problemática de las ciencias humanas se aborda así no desde la formalidad de sus procesos constructivos, sino desde la positividad de un saber cuya vigencia no queda justificada en una coherencia argumentable, ni tampoco en una pura referencia a intencionalidades o intereses previos. Este saber se presenta como un acontecimiento, un juego efectivo de afirmaciones y exclusiones cuyos elementos y reglas constitutivas es preciso analizar y comprender tanto a nivel de las relaciones entre los enunciados como de los soportes materiales / institucionales donde estas relaciones se producen y reproducen.

Es en este sentido que Foucault propone analizar los 'sistemas de formación de los discursos': no interesan ya los estados terminales de una ciencia sino esa compleja red de relaciones que prescribe las reglas de una práctica discursiva "para que ésta se refiera a tal o cual objeto, para que ponga en juego tal o cual enunciación, para que utilice tal o cual concepto, para que organice tal o cual estrategia. Definir en su individualidad singular un sistema de formación es, pues, caracterizar un discurso o un grupo de enunciados por la regularidad de una práctica".(8)

"Foucault ha descubierto que, para comprender la variedad de los tipos enunciados, el arqueólogo debla tener en cuenta la evolución

(7) "... Por lo que se refiere a la prisión, no tendría sentido limitarse a los discursos hechos sobre ella. Existen además los que provienen de la misma prisión, las decisiones, los reglamentos, que son elementos constitutivos de la prisión, del mismo funcionamiento de la prisión que tiene sus estrategias, sus discursos no formulados, sus astucias que en último término no son de nadie pero que, sin embargo, son vividas, que aseguran el funcionamiento y la permanencia de la institución Es todo lo que hay a la vez que recoger y que mostrar. Y el trabajo, a mi parecer, consiste sobre todo en hacer aparecer estos discursos en sus conexiones estratégicas en vez de constituirlos mediante la exclusión de otros discursos". M. Foucault, "Entrevista sobre la prisión: el libro y su método", en "Microfisica del poder", La Piqueta, Madrid, 1979, p. 88.

(8) M. Foucault, "La arqueologia del saber", p. 122-123. 
sistemática de un cierto número de otras prácticas discursivas: determinar, por ejemplo, quién está acreditado para producir enunciados, de qué lugar emanan éstos, qué posición ocupa el sujeto del discurso. (...) Foucault restringe su campo de investigación a esta sola cuestion: ¿a quién podemos tomar en serio, es decir, quién tiene el derecho de hablar desde una posición que presupone que lo que dice es verdadero?. $Y$ esta pregunta nos lleva directamente al sistema más general de relaciones discursivas que autoriza la formacinón de los enunciados serios y su transmision por locutores igualmente serios". (9)

La empresa arqueológica consiste justamente en rastrear los discursos y las prácticas vigentes en una época para descubrir las regularidades y normas que rigen sus procesos de distribucion, transformación, exclusión... La cuestión central refiere precisamente a esa red de mecanismos y reglas que estructuran una práctica discursiva, que definen las posiciones de los sujetos participantes y que se van permeando de un tipo de práctica a otra.

"En el análisis que se propone aqut, las reglas de formacion tienen su lugar no en la "mentalidad" o la conciencia de los individuos, sino en el discurso mismo; se imponen, por consiguiente, según una especie de anonimato uniforme, a todos los individuos que se disponen a hablar en ese campo discursivo". (10)

3) - Desde esta perspectiva, el discurso de las ciencias humanas ya no se contiene en el orden del texto, más o menos relativo a un contexto de praxis, sino que se sitúa y se despliega en una red dispersa de enunciados y estrategias que se determinan reciprocamente.

Con esto queremos decir que, al abordar el discurso clínico, el discurso carcelario o el discurso psiquiátrico, lo que se propone no es una confrontación ni una justificación epistemológica de las pretensiones de validez de sus teorías; no se trata de desarrollar una crítica del

(9) H. Dreyfus-P.Rabinow, Op. cit., pág. 103-104. (La traducción es nuestra)

(10) M. Foucault, "La arqueología del saber", pág. 102 
saber que pueda discernir entre las 'verdades' y los 'errores' en los procesos y productos del conocimiento científico; este enfoque supondría, de alguna manera, la convalidación de un orden objetivo que coincide con lo dado y constituye así el referente previo de las elaboraciones teóricas. El carácter crítico del planteo de Foucault parte de una inversión de los términos: interesa saber de qué manera los discursos pretendidamente 'serios' (11) respaldan desde su validez / verdad consensuada cierta regulación de un orden objetual / conceptual / estratégico que tiene lugar a partir de las prácticas discursivas mismas.

No hay 'errores' entonces en las formaciones discursivas. Y esto no sólo por su íntima coherencia sino por su clara adecuación a la problematización del objeto que las teorías construyen en la medida misma de su desarrollo sistemático. Lo que está en cuestión no es la verdad de las teorías ni sus criterios de validez. Lo que 'pone en foco' la crítica foucaultiana son los "efectos" de estos discursos, es decir, la producción de objetos 'problemáticos', conceptos 'verdaderos', posiciones 'válidas', estrategias 'adecuadas'.

La palabra misma "efectos" se presta a ambigüedades, o, mejor dicho, a desplazamientos. Inicialmente podríamos entender que se refiere a la incidencia de un nivel teórico propio del discurso en el plano de realización efectiva de ciertos hechos objetivos (en general, las nociones de 'información' 'aplicación', contribuyen a esta comprensión). Pero existe un nivel primario más fuerte de producción de las formaciones discursivas y es el que tiene lugar en la medida misma del surgimiento / aceptación de un discurso teórico que consolida un campo preciso de visibilidad / enunciabilidad / tratamiento de ciertas problemáticas.

(11)"Foucault considera que nuestra cultura tiene tendencia a transformar una proporción siempre creciente de nuestros actos de discurso ordinario en actos de discurso serios. Cualquier acto de discurso puede ser serio a condición de que se convoquen los procedimientos de validación necesarios, la comunidad de expertos, etc. (...) El método de justificación y de refutación es lo que confiere a los actos de discurso serios su pretensión de constituir un saber y los transforma en objetos que se pueden estudiar, repetir y transmitir. (. , 
"... La verdad no se perfila como concordancia de un pensamiento con su objeto, sino que se configura como lo que apremia a un pensamiento a pensar de una cierta manera o, según senalaremos, como cuidado, gobierno indisolublemente unido, de st y de los otros". (12)

"El problema no es saber bajo qué condiciones un enunciado sera verdadero, sino cuáles son los diferentes juegos de verdad y falsedad que se instauran y sobre quéformas. Es una critica de las veridicciones. El problema no es saber cómo un sujeto en general puede conocer un objeto en general, el problema es saber cómo los sujetos están efectivamente ligados en y por las formas de veridicción en las que se comprometen. En este caso, el problema no es el de determinar los accidentes históricos, las circunstancias extremas, los mecanismos de ilusiones o las ideologias, o la economía interna de fallas o errores lógicos que han podido producir la falsedad. El problema es el de determinar cómo un modo de veridicción, una Wahrsagen, pudo aparecer en la historia y bajo qué condiciones". (13)

El análisis prosigue entonces con el interés de desentrañar el "régimen de verdad" que rige en una sociedad en la medida en que ésta valoriza un saber, lo distribuye y refuerza a través de sus prácticas. Por

Ciertamente, las instituciones, acontecimientos políticos, prácticas y procesos económicos tienen incidencia sobre lo 'serio' de un discurso y por supuesto los sujetos individuales deben hablar de los objetos reconocidos por el conjunto de la comunidad científica a la cual pertenecen, si quieren ser tomados en serio; pero las normas de seriedad no están determinadas ni por las relaciones reales o primarias, ni por las relaciones segundas o reflexivas, sino por la manera en que las prácticas discursivas disponen estas relaciones. (...). Lo que hace que un acto de discurso sea serio, y tenga entonces valor de enunciado, es el lugar que ocupa en la red de otros actos de discurso serios y nada más". (Dreyfus y Rabinow, Op. Cit, pág. 76 ss.)

(12) A. Gabilondo, Op. cit., pág. 182. (En este texto hace referencia a E. Ewald, "La fin d'un monde", pág. 32).

(13) T. Abraham, "Los senderos de Foucault", Ed. Nueva Visión, Bs. As. 1989, pág. 102-103. 
consiguiente, hablar de los "efectos de verdad" de los discursos supone entender que estas verdades no se juegan fundamentalmente en el orden del pensar sino en la acción misma de estos conceptos en la realidad institucional.

Es en este marco en el que Foucault propone reconsiderar las relaciones entre verdad y poder:

"Lo importante, creo, es que la verdad no está fuera del poder, ni sin poder (no es, a pesar de un mito, del que seria preciso reconstruir la historia y las funciones, la recompensa de los espiritus libres, el hijo de largas soledades, el privilegio de aquellos que han sabido emanciparse). La verdad es de este mundo; está producida aqut gracias a múltiples imposiciones. Tiene aqui efectos reglamentados de poder. Cada sociedad tiene su régimen de verdad, su "politica general de la verdad": es decir, los tipos de discurso que ella acoge y hace funcionar como verdaderos; los mecanismos y las instancias que permiten distinguir los enunciados verdaderos ofalsos, la manera de sancionar unos y otros; las técnicas y los procedimientos que son valorizados para la obtención de la verdad; el estatuto de aquéllos encargados de decir qué es lo que funciona como verdadero. " (14)

4. En las obras posteriores de Foucault -"Vigilar y Castigar", "Historia de la sexualidad"- la arqueología da paso a una genealogía del sujeto moderno a través del análisis de las relaciones saber/poder.

Foucault plantea claramente los límites de una concepción jurídica, negativa, del poder según la cual éste "es esencialmente la regla, la ley, la prohibición, lo que marca un límite entre lo permitido y lo prohibido". (15)

Al abandonar esta representación del poder, e intentar abordarlo en sus mecanismos "positivos" o "productivos", el análisis descubre las

(14) M. Foucault, "Verdad y poder, en "Microfisica del poder", La Piqueta, pág. 187.

(15) M. Foucault, "Las redes del poder", Ed. Almagesto, Bs. As. 1991, pág.9. 
formas de sujeción tal como se ejercen, en sus técnicas y modalidades específicas: la disciplina (tecnología de control de la vida de los individuos) y la regulación social (bio-poder, control de la vida de la población).

Estos mecanismos, que van consolidando a partir del siglo XIX una "sociedad disciplinaria." (16), están fuertemente conectados con los instrumentos de saber de la medicina, la psiquiatría, la psicología, la pedagogía... El nivel de problematización por el que el hombre se convierte en objeto de atención y cuidado hace posible la vinculación de instituciones, poderes y saberes a través de mecanismos precisos de clasificación y control. Los 'discursos serios', en tanto son reconocidos como tales, intervienen en el ordenamiento social haciendo posible el funcionamiento de un poder básicamente productivo. Es en el ámbito de las ciencias humanas, entendidas como 'prácticas discursivas' , donde se desarrollan las posiciones, objetivaciones y conceptos que permiten construir un modelo de disciplina y normalización.

"En una sociedad como la nuestra, pero en el fondo en cualquier sociedad, relaciones de poder múltiples atraviesan, caracterizan, constituyen el cuerpo social; y estas relaciones de poder no pueden disociarse, ni establecerse, ni funcionar, sin una producción, una acumulación, una circulación, un funcionamiento del discurso. (...) El poder no cesa de preguntarnos, de indagar, de registrar, institucionaliza la pesquisa de la verdad, la profesionaliza, la recompensa. En el fondo tenemos que producir verdad igual que tenemos que producir riquezas. Por otro lado también estamos sometidos a la verdad en el sentido en que la verdad hace ley, elabora el discurso verdadero que, al menos en parte, decide, transmite, empuja efectos de poder. Después de todo, somos juzgados, condenados, clasificados, obligados a competir, destinados a vivir de un cierto modo o a morir en función de discursos verdaderos que conllevan efectos especificos de poder". (17)

(16) Cf. "Le panoptisme", en M. Foucault, "Surveiller et Punir", Gallimard, 1975.

(17) M. Foucault, "Verdad y poder", en "Microfisica del poder", pág. 187. 
5. Al entender que las interacciones que tienen lugar en los medios no - discursivos no representan el contexto en el interior del cual se ubica el sentido del discurso, sino que constituyen elementos que son retomados y transformados por las prácticas discursivas mismas, es preciso reconsiderar el significado de la distinción teoría-práctica en el ámbito de las problemáticas que involucran al hombre.

El entrecruzamiento de conceptualizaciones, opciones y roles que inicialmente señalábamos como determinando el campo de 'visibilidad' de una disciplina, en una época, se descubre ahora como un diagrama de relaciones de fuerza que da lugar a un cierto funcionamiento teórico institucional y que configura las condiciones históricas efectivas de constitución de los sujetos.

Las ciencias humanas perfilan así un ordenamiento del espacio de los individuos por el cual éstos reconocen e interpretan sus márgenes de acción. Los procesos discursivos no tienen entonces un dinamismo puramente 'teórico' sino que forman parte de un conjunto de prácticas en las que se configura el juego de posibilidades e imposibilidades en una sociedad. En este sentido podemos leer la afirmación de Deleuze: "Estamos viviendo de una nueva manera las relaciones de teoría y práctica.(...) La práctica es un conjunto de conexiones de un punto teórico con otro, y la teoría un empalme de una práctica con otra. (...) No existe ya la representación, no hay más que acción, acción de teoría, acción de práctica en relaciones de conexión o de redes." (18)

Esta posibilidad de pensar la teoría y la práctica desde la materialidad de sus relaciones efectivas, permite un nuevo movimiento de distanciación y transformación (19). El diagnóstico de nuestro presente lo descubre como un sistema de racionalidad que se ejerce a

(18) G. Deleuze, en diálogo con M. Foucault, "Los intelectuales y el poder", en "Microfísica del poder", pág. 77-78.

(19) Al final de una conferencia, cuando se interroga a Michel Foucault sobre el poder que él mismo ejerce a través de su saber, responde situando una vez más el trabajo teórico en una red de relaciones de fuerza donde el ejercicio mismo del discurso produce efectos de movilidad, distanciación, transformación. (Cf. "Las redes del poder", pág. 29-30). 
través de dispositivos y técnicas de poder precisos. Frente a los discursos que enhebran estas prácticas racionales, la tarea crítica no pasa tanto por una exigencia de legitimación de sus reglas y contenidos sino por una reconstrucción de las problematizaciones que impulsaron este modo de objetivación de los individuos. Ahora bien, al señalar los vectores, orientaciones, jerarquizaciones y movimientos de este diagrama social, se descubren también los intersticios de la red, desde los cuales se cuestiona su ordenamiento, sus fronteras. Se abre entonces el espacio de un juego teórico-práctico en el que se intente ver cómo y hasta qué punto es posible pensar de otra manera .

La arqueología del saber pasa a constituir así el umbral de una nueva problematización.

\section{RESUMEN}

En este trabajo proponemos reconsiderar algunas problemáticas que se hacen presentes en la reflexión, en torno a las ciencias humanas a partir de la categoría foucaultiana de "practicas discursivas".

$\mathrm{La}$ arqueología del saber analiza el archivo de las formaciones discursivas que están presentes en la genealogía del sujeto moderno, señalando sus mecanismos de apropiación y exclusión, recorriendo el diagrama de relaciones discursivas y no-discursivas que regula el régimen de verdad de una sociedad. Mas acá de la discusión acerca de la validez de las teorías científicas, los "discursos serios" se descubren como acontecimientos que producen "efectos de verdad" a nivel de la interacción social y de la vida de los individuos.

Se plantea así desde una nueva perspectiva el problema del saber-poder en la sociedad moderna y se resignifican los términos de la relación teoríapráctica en el ámbito de las ciencias humanas. 